\title{
Plasma membrane-associated sialidase (NEU3) regulates progression of prostate cancer to androgen-independent growth through modulation of androgen receptor signaling
}

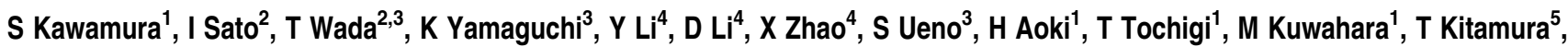 \\ K Takahashi ${ }^{3,6}$, S Moriya ${ }^{3,6}$ and T Miyagi, ${ }^{\star 3,6}$
}

Prostate cancers generally become androgen-independent and resistant to hormone therapy with progression. To understand the underlying mechanisms and facilitate the development of novel treatments for androgen-independent prostate cancer, we have investigated plasma membrane-associated sialidase (NEU3), the key enzyme for ganglioside hydrolysis participating in transmembrane signaling. We have discovered NEU3 to be upregulated in human prostate cancer compared with non-cancerous tissue, correlating with the Gleason score. NEU3 silencing with siRNA in prostate cancer PC-3 and LNCaP cells resulted in increased expression of differentiation markers and in cell apoptosis, but decrease in $\mathrm{Bcl}-2$ as well as a progression-related transcription factor, early growth response gene (EGR-1). In androgen-sensitive LNCaP cells, forced overexpression of NEU3 significantly induced expression of EGR-1, androgen receptor (AR) and PSA both with and without androgen, the cells becoming sensitive to androgen. The NEU3-mediated induction was abrogated by inhibitors for PI-3 kinase and MAP kinase and more specifically by their silencing in the absence of androgen, being confirmed by increased phosphorylation of AKT and ERK1/2 in NEU3 overexpressing cells. NEU3 siRNA introduction caused reduction of cell growth of an androgen-independent PC-3 cells in culture and of transplanted tumors in nude mice. These data suggest that NEU3 regulates tumor progression through AR signaling, and thus be a potential tool for diagnosis and therapy of androgen-independent prostate cancer.

Cell Death and Differentiation (2012) 19, 170-179; doi:10.1038/cdd.2011.83; published online 17 June 2011

Androgen receptor (AR) signaling has a central role in prostate cancer pathogenesis and progression, and androgen withdrawal is the main therapeutic options for treatment of advanced cases. ${ }^{1,2}$ However, many prostate cancers eventually become androgen-independent, and this results in renewed growth and spread. The mechanism underlying the initial survival and subsequent growth of ablation-resistant prostate cancer cells is not fully characterized. However, several lines of evidence point to activation of AR signaling under androgen-independent conditions. Likely, mechanisms so far proposed include $A R$ mutations, allowing receptors to be activated by new ligands, ${ }^{3} A R$ gene overexpression causing AR oversensitive to low concentrations of androgen $^{4,5}$ and cross-stimulation of AR signaling by other growth factors. ${ }^{6,7}$ It has been previously shown that early growth response gene $1(E G R-1)$ is an important transcription factor involved in promoting prostate cancer progression, ${ }^{8,9}$ its overexpression enhancing androgen-independent growth of prostate cancer cells by modulating androgen signaling through increased AR nuclear transition. ${ }^{10}$ To facilitate the search for new effective therapeutic targets for androgen- independent prostate cancer, molecular mechanisms should be elucidated from various aspects. In this study, we focused on a human sialidase, a glycosidase catalyzing the removal of sialic acids from glycolipids and participating transmembrane signaling, ${ }^{11,12}$ because the enzyme has been found to be upregulated in several human cancers and to suppress apoptosis of cancer cells. ${ }^{13-15}$

Alterations in glycosylation occur during tumorigenesis, and aberrant sialylation in particular has been implicated in the malignant phenotype with reference to metastatic potential and invasiveness. ${ }^{16-18}$ Mammalian sialidase is the key enzyme for control of cellular sialic acid contents, through catalyzing the initial step in degradation of glycoproteins and glycolipids. Four types of human sialidases have been identified and characterized to date, designated as NEU1, NEU2, NEU3 and NEU4. ${ }^{11,12}$ They differ in major subcellular localization and enzymatic properties including substrate specificity, and each has been found to have a unique functional role depending on its particular properties other than lysosomal catabolism. They behave in different manners during carcinogenesis. ${ }^{19}$ Of the four types of sialidase,

\footnotetext{
${ }^{1}$ Department of Urology, Miyagi Cancer Center, Natori, Japan; ${ }^{2}$ Division of Pathology, Miyagi Cancer Center Research Institute, Natori, Japan; ${ }^{3}$ Division of Biochemistry, Miyagi Cancer Center Research Institute, Natori, Japan; ${ }^{4}$ Department of Pathophysiology, Prostate Diseases Prevention and Treatment Research Center, Jilin University of Medical Sciences, Changchun, PRC, China; ${ }^{5}$ Division of Cellular Therapy, Institute of Medical Science, University of Tokyo, Tokyo, Japan and ${ }^{6}$ Division of Cancer Glycosylation Research, Institute of Molecular Biomembrane and Glycobiology, Tohoku Pharmaceutical University, Sendai, Japan

*Corresponding author: T Miyagi, Division of Cancer Glycosylation Research, Institute of Molecular Biomembrane and Glycobiology, Tohoku Pharmaceutical University, 4-4-1 Komatsushima, Aoba-ku, Sendai 981-8558, Japan. Tel: + 8122727 0157; Fax: + 8122275 2013; E-mail: tmiyagi@ tohoku-pharm.ac.jp Keywords: sialidase; prostate cancer; androgen receptor; EGR-1; PSA

Abbreviations: AR, androgen receptor; EGR-1, early growth response gene 1; DHT, dihydrotestosterone; EGFR, epidermal growth factor receptor Received 03.1.11; revised 06.5.11; accepted 12.5.11; Edited by B Zhivotovsky; published online 17.6.11
} 
plasma membrane-associated sialidase, NEU3, is unique in specifically hydrolyzing gangliosides, thought to participate in cell differentiation and transmembrane signaling. ${ }^{11,12}$ In fact, recent observations have suggested an involvement of NEU3 in regulation of signal transduction through ganglioside modulation and interaction with some signaling molecules. NEU3 is upregulated in various human cancers, including colon, ${ }^{13}$ renal $^{14}$ and ovarian cancers, ${ }^{15}$ and contributes to the expression of malignant properties, including suppression of apoptosis by activating EGF receptor signaling, ${ }^{20}$ and promotion of cell invasion and motility by affecting integrinmediated signaling in the extracellular matrix in colon cancer cells ${ }^{21}$ and by activating IL-6-mediated signaling via the PI3 kinase (PI3K)/Akt cascade in renal cancer cells. ${ }^{14}$ Our present data show that upregulation of NEU3 is also observed in prostate cancer, activating AR signaling by increasing EGR-1, $A R$ and $P S A$ expression, possibly via epidermal growth factor receptor (EGFR) family activation, and causing androgen-independent proliferation of the cancer cells.

\section{Results}

NEU3 is upregulated in human prostate cancer. Relative mRNA levels of NEU3 measured by quantitative RT-PCR were $4.2 \pm 2.5$ and $1.2 \pm 1.3(P<0.0001)$ for 34 cancer and 35 non-cancerous samples, respectively, indicating that NEU3 in tumor specimens is significantly upregulated as compared with non-tumor prostate tissues (Figure 1a). The sialidase activity toward gangliosides showed a tendency to be increased in tumors, even in the limited number of specimens examined (Figure 1b). The mRNA levels positively correlated with the prostate cancer tissue grade, as assessed by Gleason score $(P<0.0001)$ as shown in Figure 1c. Consistent with these data, immunohistochemistry with anti-NEU3 monoclonal antibody exhibited strong positive staining in prostate cancer tissues with a high Gleason score, whereas non-tumor prostate tissues were almost negative (Figure 1d).

NEU3 modulates expression of differentiation- and apoptosis-related molecules in human prostate cancer cell lines. To determine whether the increased NEU3 level in prostate cancer was reproducible in vitro, we analyzed two human prostate cancer cell lines, PC-3 and LNCaP. Androgen-insensitive and apoptosis-resistant PC-3 cells expressed a higher level of NEU3 than androgen-sensitive LNCaP cells, with reference to both sialidase activity and the mRNA level (Figure 2a). To examine whether the increased NEU3 expression affects promotion of prostate cancer progression, we then prepared NEU3 overexpressing cells by retrovirus transfection and NEU3 silencing cells with NEU3 targeting siRNA. The sialidase activities after NEU3 overexpression were $97.7 \pm 14.1 \mathrm{U} / \mathrm{mg}$ and $104.5 \pm 12.6 \mathrm{U} / \mathrm{mg}$ protein in PC-3 and LNCaP cells, respectively. After NEU3 silencing, in comparison with control siRNA, the relative mRNA levels were $17.7 \pm 7.0 \%$ and $19.6 \pm 8.5 \%$, and the sialidase activities were $1.33 \pm 0.51 \mathrm{U} / \mathrm{g}$ and $0.28 \pm 0.11 \mathrm{U} / \mathrm{g}$ protein in PC-3 and LNCaP cells, respectively.
When the cell lines were treated with $5 \mathrm{mM}$ sodium butyrate for $48 \mathrm{~h}$, endogenous NEU3 was downregulated and the cells underwent differentiation and apoptosis. On the other hand, NEU3 overexpression resulted in suppression of apoptosis in both PC-3 and LNCaP cells, and furthermore, mock-transfected PC-3 cells seemed to be more resistant than that of LNCaP cells having a lower level of endogenous NEU3, as assessed by FACS analysis with AnnexinV Fluolostaining (Roche Diagnostics, Penzberg, Germany; Figure 2b). In the both cells, ectopic expression of NEU3 caused decreased mRNA levels for keratin 17 and IL24 differentiation markers, and increased the level for antiapoptotic protein Bcl-2. Knockdown of NEU3 led to essentially the opposite results (Figure $2 \mathrm{c}$ ). These results indicate that NEU3 upregulation in prostate cancer might cause suppression of cell differentiation and apoptosis.

NEU3 enhances expression of AR signaling-related molecules. We next assessed expression levels of $A R$ signaling-related molecules to observe whether NEU3 is involved in AR signaling pathway. NEU3 enhanced mRNA production for the progression-related transcription factor EGR-1 in both lines, and increased levels for AR and PSA in LNCaP cells, whereas NEU3 knockdown lowered AR and PSA mRNA expression (Figure 3a). EGR-1 has been described to have a crucial role in prostate cancer progression and levels correlate positively with the tumor grade. ${ }^{8,9}$ In addition to suppression of cell differentiation and apoptosis as shown in Figure 2, NEU3 might cause activation of the AR signaling pathway. When protein levels of $A R$ and PSA were analyzed by immunoblotting in LNCaP cells cultured with charcol/dextran-stripped serum, the levels were increased not only by dihydrotestosterone (DHT) also by NEU3 even without DHT, and treatment with an anti-androgen drug bicalutamide returned these proteins to their basal levels (Figure 3b). On the other hand, NEU3 silencing significantly reduced DHT-dependent increase in EGR-1, AR and PSA protein levels as well as basal levels of $A R$ and PSA (Figure $3 c$ ). These results indicate that NEU3 promotes functional activation of androgen signaling in LNCap cells both in the presence and absence of androgen. To analyze glycolipid changes as a result of modulation of NEU3 expression, glycolipids extracted from the cells were subjected to thin-layer chromatography, because of special preference of the sialidase to gangliosides. As shown in Figure 3d, NEU3 overexpression yielded an increase in lactosylceramide with a slight Gb3 increase as compared with control, but there was no detectable change before and after NEU3 silencing. We also tried to detect a small change of cell surface gangliosides by FACS analysis with antibodies against GD1a, GM2, GM1 (Seikagaku Biobusiness, Tokyo, Japan) and GD2 (Upstate, Lake Placid, NY, USA), as a previous report suggested possible existence of these gangliosides in the cells. ${ }^{22}$ However, all the antibodies failed to detect any significant change in either NEU3 overexpressing or silencing cells (data not shown).

NEU3 activates AR signaling via PI3K and MAP kinase (MAPK) pathways. To gain insights into the signaling pathway involved in the NEU3-mediated induction of these 

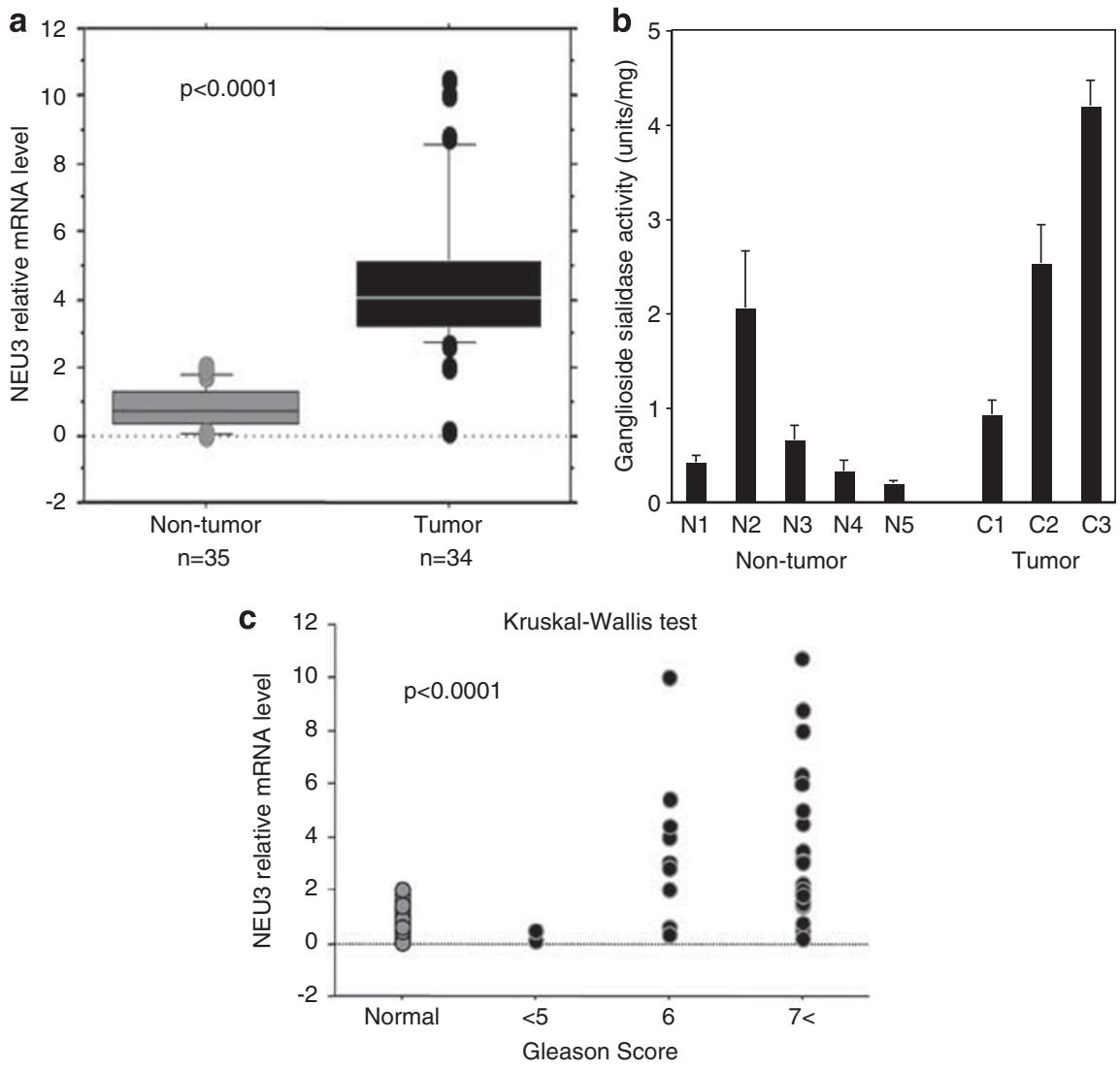

d

Normal
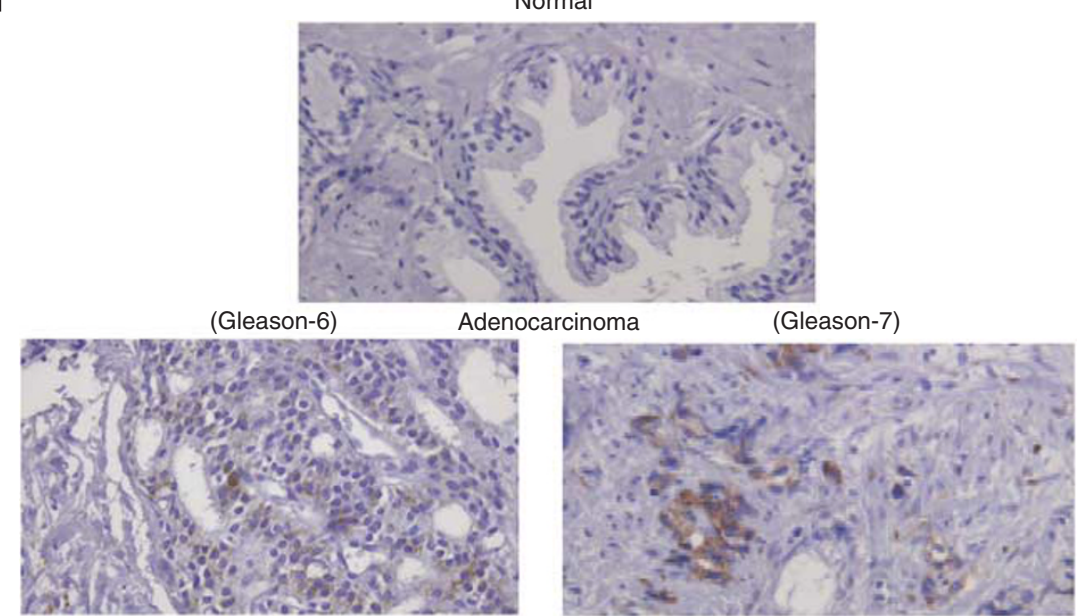

Figure 1 Upregulation of NEU3 in human prostate cancer. (a) Relative mRNA levels of NEU3 assessed by quantitative RT-PCR for 34 cancer and 35 non-cancerous samples. Box-and-whisker plots were used for statistical analysis. (b) Sialidase activity levels of cancer and non-cancerous samples assessed by fluorometric highperformance liquid chromatography with malononitrile. (c) Co-relationship between NEU3 level and prostate cancer grading, Gleason score. The data were statistically analyzed using the Kruskal-Wallis test. (d) Immunohistochemistry with anti-NEU3 monoclonal antibody for prostate cancer tissues with the high Gleason score and non-tumor prostate tissues

AR-related proteins, specific inhibitors of PI3K (LY294002) and MAPK (PD098059) pathways were used. ${ }^{23}$ Treatment with LY294002 inhibited NEU3-induced EGR-1 and AR elevation, whereas PD098059 abolished the PSA increase (Figure 4a). To be more convincing, the siRNAs for PI3K p110 $\alpha$ and for MAPKK (MEK1/2) were separately used to block specifically these expressions. In the cells of PI3K knocking down, NEU3-mediated AR induction was inhibited, and the cells bearing MEK1 knockdown showed significantly lowered PSA expression as expected (Figure 4b). These results indicate that NEU3 induces and activates EGR-1 and AR through the PI3K pathway and PSA through the MAPK pathway, in an androgen-depleted environment. Consistent with these data, NEU3 significantly promoted 
a

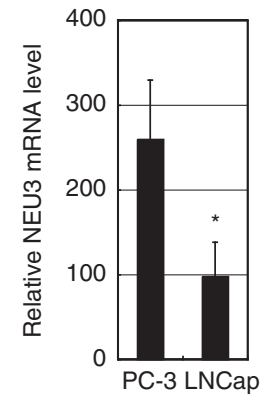

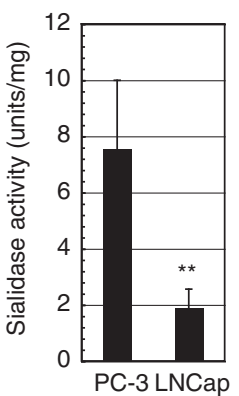

b
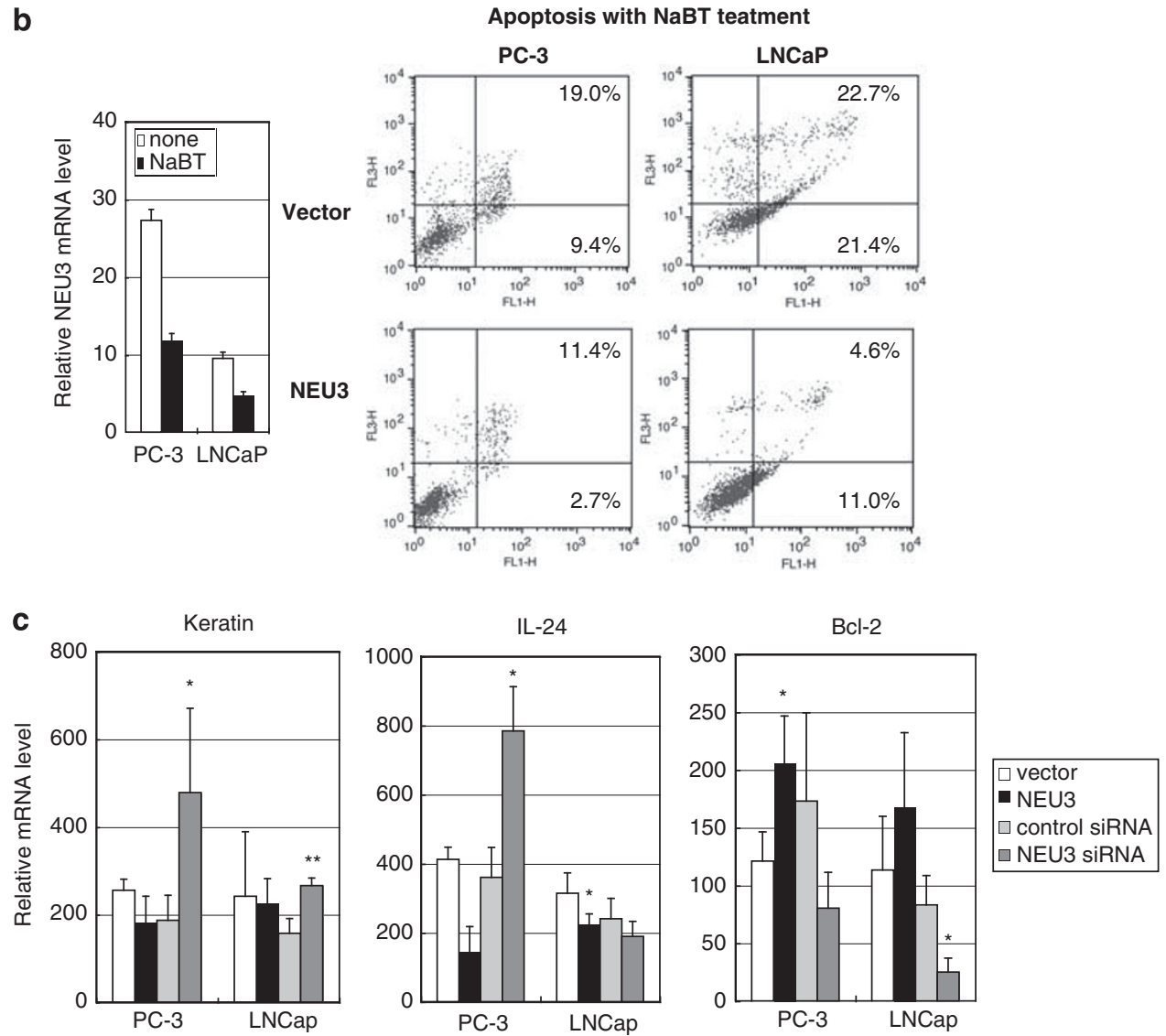

Figure 2 Altered expression of differentiation- and apoptosis-related molecules by NEU3 in human prostate cancer cell lines. (a) NEU3 expression in mRNA and sialidase activity levels in $\mathrm{LNCaP}$ cells and PC-3 cells. The data given are mean values from five to seven experiments \pm S.D. $P$-values ( ${ }^{\star} P=0.015$, ${ }^{\star \star} P=0.0003$ ) were evaluated by the Student's t-test, and compared with PC-3 cells. (b) Apoptosis induction of PC-3 and LNCaP cells by treatment with sodium butyrate, and apoptosis suppression by NEU3 overexpression. Apoptosis was assessed by FACS analysis with AnnexinV. Results are representative of three independent experiments. (c) Measurement of mRNA levels of keratin 17 and IL24, and Bcl-2 in NEU3-silencing or -overexpressing cells. ${ }^{*} P<0.05,{ }^{* *} P<0.01$ compared with control siRNA or vector by the Student's $t$-test

phosphorylation of AKT and ERK in the same condition (Figure 4c). To elucidate further how NEU3 affects these pathways, upstream molecules were investigated. We previously demonstrated that NEU3 promotes phosphorylation of EGFR by enhancing dimerization in HeLa cells. ${ }^{20}$ It has also been reported that the EGFR family modulates AR signaling and contributes androgenindependent tumor progression. ${ }^{24-28}$ Expression levels of EGFR family were therefore evaluated as possible targets for NEU3 (Figure 4d). NEU3 was found to increase EGFR and ERBB2 in mRNA and protein levels together with $A R$ and PSA elevation in LNCaP cells under androgendeficient conditions, although they behaved differently in the presence of DHT. Phosphorylation level of ERBB2 was enhanced on a parallel with increase in the protein level, and DHT addition caused marked reduction of the phosphorylation. EGFR phosphorylation was not detectable under the condition as expected, but addition of EGF led to the higher phosphorylation level in NEU3 overexpressing cells than the control cells (Figure 4e). Interestingly, ERK phosphorylation was always augmented by NEU3, independent of androgen or EGF. These results suggest 
a

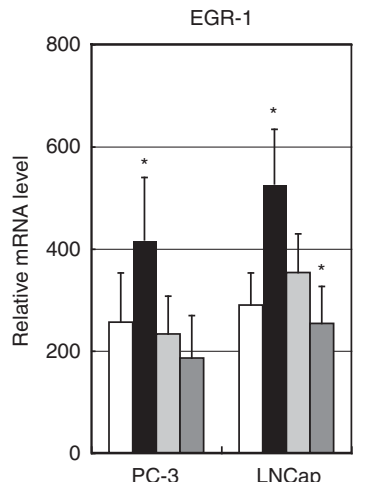

b

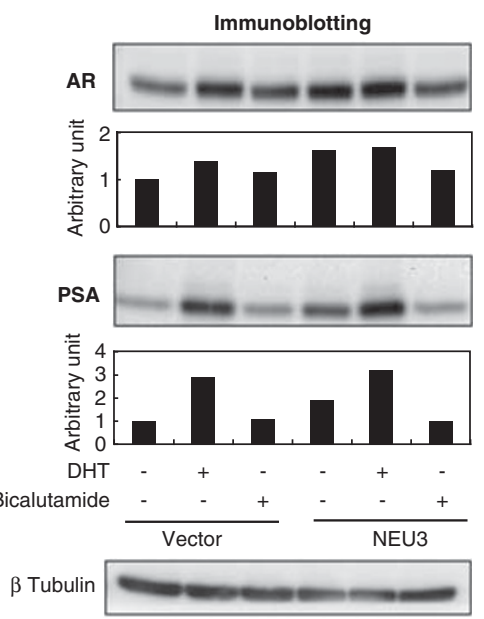

AR

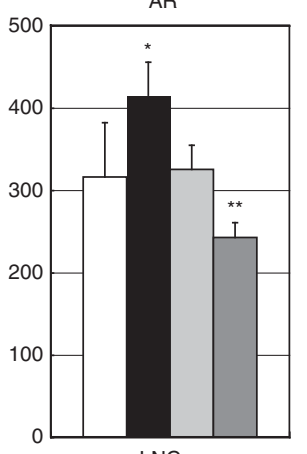

PSA

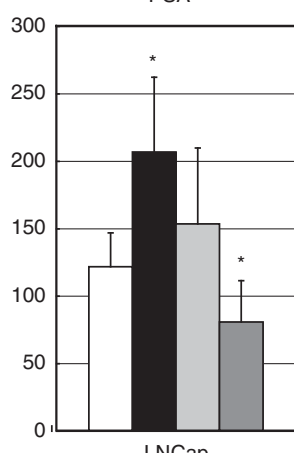

$\square$ vecto

IEU3

$\square$ control siRNA

$\square$ NEU3 SiRNA c

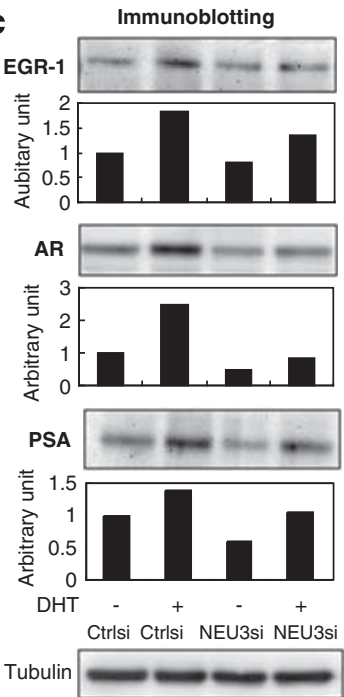

d

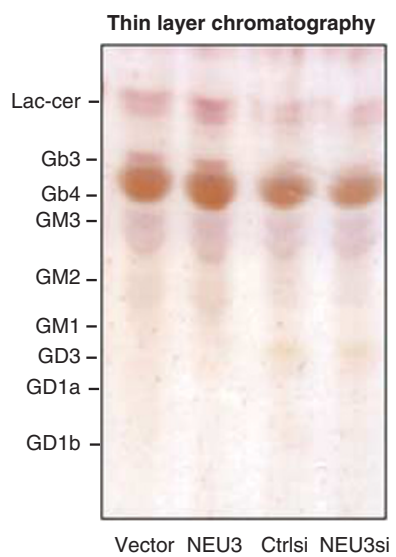

Figure 3 Altered expression of AR signaling-related molecules by NEU3 in LNCaP cells. (a) Altered mRNA expression of AR signaling-related molecules in NEU3silencing or -overexpressing cells. ${ }^{*} P<0.05,{ }^{* *} P<0.01$ compared with control siRNA or vector. EGR-1, AR and PSA levels were assessed by RT-PCR as described in Materials and Methods. (b) Protein levels of AR and PSA in the presence of DHT or bicalutamide in NEU3-overexpressing and control cells as assessed by immunoblotting with respective antibodies. (c) Protein levels of EGR-1, AR and PSA in the presence or absence of DHT in NEU3-silencing or control cells as assessed by immunoblotting. In $\mathbf{b}$ and $\mathbf{c}$, the results are representative of three independent experiments. Quantitative data are presented as values relative to those in the cells treated with the vector or siRNA control in the absence of DHT and bicalutamide. (d) Thin-layer chromatography of glycolipids from NEU3-overexpressing and -silencing cells. After development, the glycolipids were visualized with orcinol- $\mathrm{H}_{2} \mathrm{SO}_{4}$. Results are representative of three independent experiments

that NEU3 activates AR signaling probably via induction of EGFR and/or ERBB2 under androgen-deficient conditions.

NEU3 potentiates DHT-dependent cell growth. To determine the effects of NEU3-mediated activation of AR signaling on cell proliferation, NEU3 transfectants were cultured in the presence of $5 \mathrm{nM}$ DHT or $5 \mu \mathrm{M}$ antiandrogen bicalutamide (Figure 5a). DHT-induced LNCaP cell proliferation was remarkably increased as compared with the case with the vector control, and even $1 \mathrm{nM}$ DHT caused significant stimulation of cell growth (data not shown), indicating that NEU3 enhances sensitivity to androgen, presumably via increase in numbers of ARs. Furthermore, interestingly, NEU3 stimulated cell growth even in the presence of bicalutamide. We do not know the mechanism of the NEU3 effects, but it has been suggested that increased AR expression converts even antagonists to weak agonists. ${ }^{1,2}$ When AR were silenced to $10-15 \%$ the level of control cells, the cell growth rate was significantly reduced as compared with the cells having control AR shown in Figure 5a, implying AR dependence (Figure $5 \mathrm{~b}$ ). In $A R$-silenced cells, however, growth stimulation by NEU3 gradually occurred with an evident response to DHT after 3 days, even when decreased level of AR was sustained (to less than $15 \%$ of the control level for 5 days), possibly due to compensatory activation of AR signaling. To examine the possibility whether $A R$ silencing gives any influence on expression levels of $A R$ signaling related molecules, the protein and mRNA levels were evaluated in $A R$ silencing LNCaP cells (Figure $5 \mathrm{c}$ ). Under conditions in which AR and PSA was reduced profoundly, $A R$ silencing stimulated ERK phosphorylation (left panel) and increased NEU3 and EGR-1 expression level (right panel), suggesting that the compensatory activation of AR signaling occurs as the results of NEU3 and EGR-1 elevation at the endogenous level. Reduced AR expression, including that following castration, thus might bring about NEU3 elevation, and finally end up with increased $A R$ and continued cell growth. Taken together, NEU3 upregulation in prostate cancer 


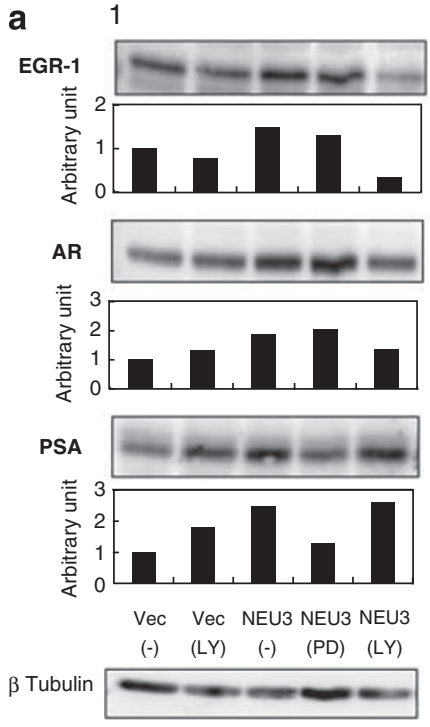

C

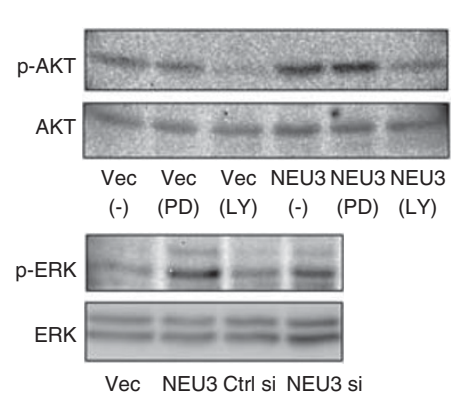

2
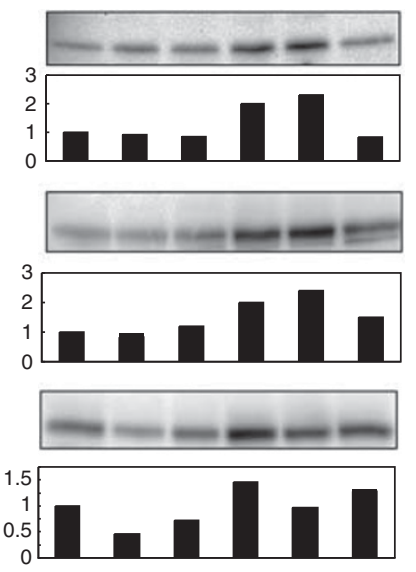

Vec Vec Vec NEU3 NEU3 NEU3

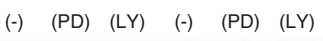

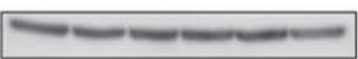

d
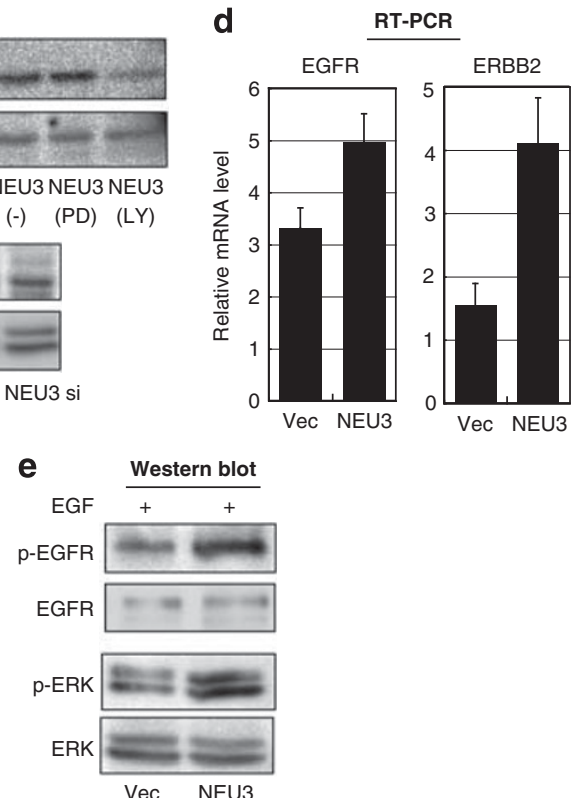

b
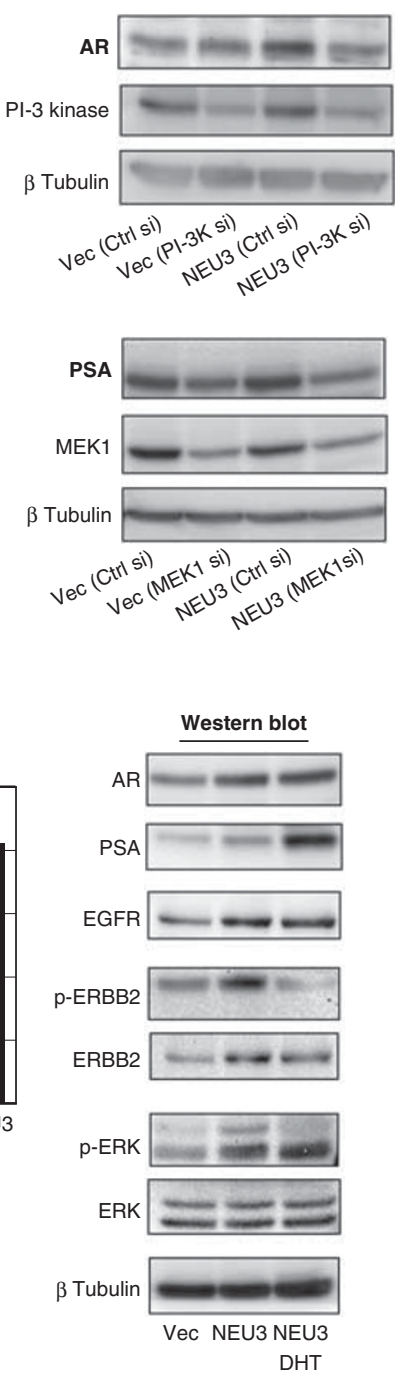

Figure 4 Activation of AR signaling by NEU3 via PI3K and MAPK pathway in LNCaP cells. (a) Effects of specific inhibitors of PI3K (LY294002) and MAPK (PD098059) on NEU-3-mediated increase in EGR-1, AR and PSA protein levels, as assessed by immunoblotting. The results are two representatives of four independent experiments. Quantitative data are presented as described in Figure 3. (b) Effects of silencing of PI3K and MAPKK on NEU-3-mediated increase in AR and PSA. The siRNAs for PI3K p110 $\alpha$ and for MAPKK (MEK1/2) were separately introduced to block specifically these kinase expressions. Similar results were obtained in two independent experiments. (c) Increased phosphorylation of AKT and ERK by NEU3. (d) Increased expression of EGFR and ERBB2 by NEU3 as evaluated by RT-PCR (left panel) and immunoblotting (right panel), respectively. Enhanced phosphorylation of ERBB2 and ERK by NEU3 under androgen-depleted condition (left panel). (e) Phosphorylation of EGFR and ERK by NEU3 in the presence of EGF

indeed causes elevation of EGR-1, AR and PSA expression by activating AKT and ERK at least partly through EGFR family activation, leading to increasing sensitivity to low concentration of androgen and to progressive cell growth, under androgen-deficient conditions (Figure 5d). NEU3 upregulation may trigger off activation of AR pathway probably via EGFR family followed by ERK activation, leading to transcriptional activation of NEU3 itself by SP1 transcription factor in positive feedback loop, as $\mathrm{Sp} 1$ is phosphorylated by ERK and NEU3 is an SP1 target gene. ${ }^{29}$
NEU3 gene silencing with siRNA suppresses prostate cancer growth. To investigate the effects of siRNAmediated NEU3 silencing on prostate cancer growth and survival, NEU3 siRNA was introduced into the cells and xenografts. Figure $6 a$ shows that NEU3 silencing caused reduction in the number of viable cells of the both prostate cancer cell lines as measured by MTT assays, whereas they continued to proliferate after treatment with a control siRNA treatment. In LNCaP cells, NEU3 silencing brought about decreased expression of EGR-1, AR and PSA as shown in 


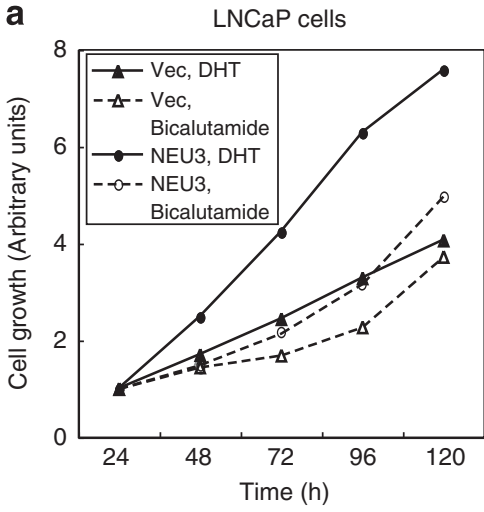

C

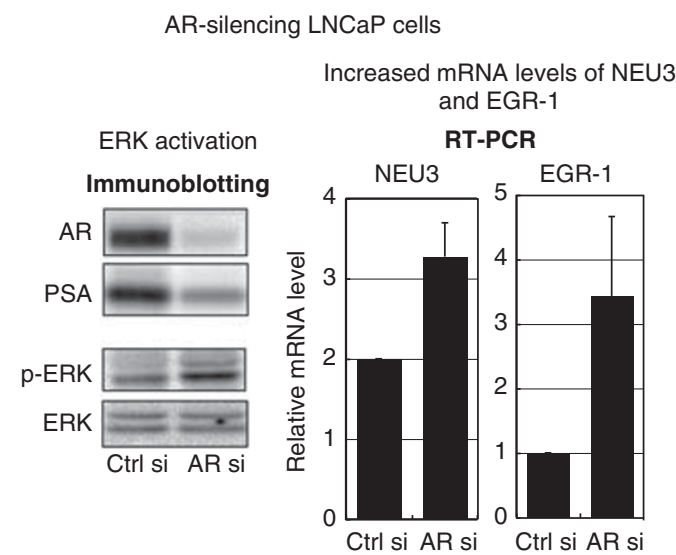

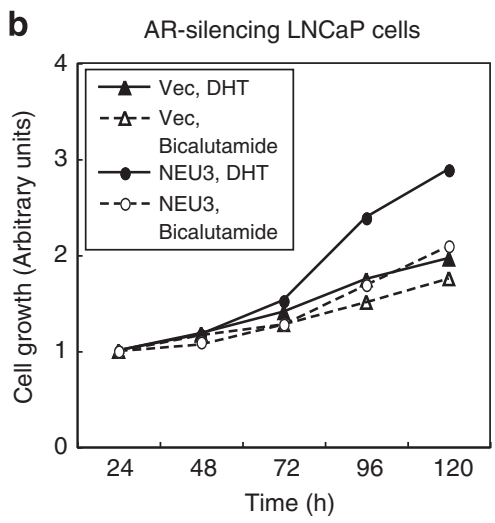

d

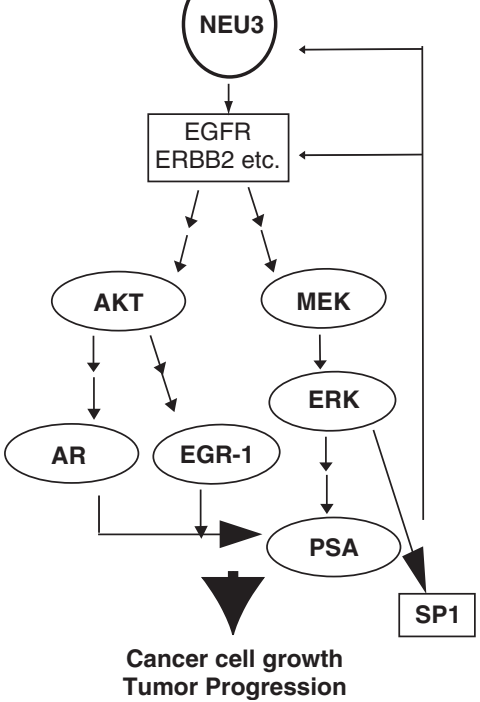

Figure 5 Acceleration of androgen-dependent cell growth by NEU3 in LNCaP cells. An increase in DHT-induced cell proliferation by NEU3 in LNCaP cells (a) and in AR silencing LNCaP cells (b), as determined by MTT assays. (c) Increased expression of NEU3 and EGR-1 (in RT-PCR, right panel) in AR silencing cells under conditions in which AR and PSA were reduced profoundly (in immunoblotting, left panel). Stimulated ERK phosphorylation seen by AR silencing (left panel). (d) Proposed model of a possible activation pathway of AR signaling regulated by NEU3 under androgen-deficient conditions

Figure 3c, contributing to the suppression of cell growth. The NEU3 siRNA-treated cells showed apoptosis at 72-96 $\mathrm{h}$ as assessed by a TUNEL assay (data not shown), as we previously described in HeLa cells. ${ }^{20}$ To further test the utility of NEU3 siRNA as a therapeutic agent for prostate cancer in vivo, androgen-independent PC-3 cells, which feature higher NEU3 expression and stronger apoptosis-resistance than LNCaP cells, were implanted subcutaneously into nude mice. When the tumor volume reached at least $70-80 \mathrm{~mm}^{3}$ at 3-4 weeks later, 10 mice for each group were administered NEU3 siRNA-atelocollagen complexes or atelocollagen ${ }^{30,31}$ alone into the tumors. The volume of tumors injected with NEU3 siRNA was statistically less than that of control tumors, which continued to increase in size, and the $500 \mathrm{pmol}$ dose siRNA was more effective than $250 \mathrm{pmol}$ (Figure 6b). Immunohistological examination revealed suppressed NEU3 protein expression as well as lowered Ki-67 labeling together with more apoptotic cells in the siRNA-effective cases, whereas mitosis was observed frequently on HE staining and the Ki-67 labeling index was higher in control tumors (Figure $6 \mathrm{c}$ ). These results indicate that the tumor reduction is probably due to inhibition of NEU3 expression.

\section{Discussion}

In this study, we demonstrated NEU3 expression to be markedly increased in human prostate cancers, and that upregulation caused suppression of cell differentiation and apoptosis. We also found that NEU3 activated AR signaling by increasing mRNA and protein levels of EGR-1, AR and PSA in $\mathrm{LNCaP}$ cells, to levels sufficient to maintain androgenindependent growth. ${ }^{1}$ Silencing of the NEU3 gene with an siRNA significantly inhibited prostate cancer cell growth, and administration of siRNA-atelocollagen complexes suppressed NEU3 protein expression, resulting in a decreased growth rate of nude mouse-transplanted tumors.

Our previous observations ${ }^{20}$ revealed that NEU3 silencing induces apoptosis with no specific stimuli, accompanied by decreased $\mathrm{Bcl}-\mathrm{xL}$ and increased Bax mRNA levels as well as 


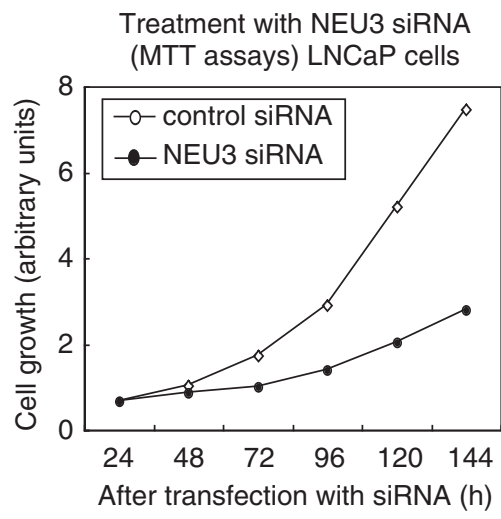

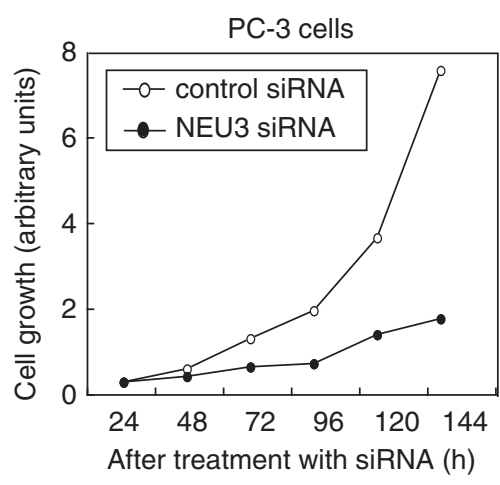

b

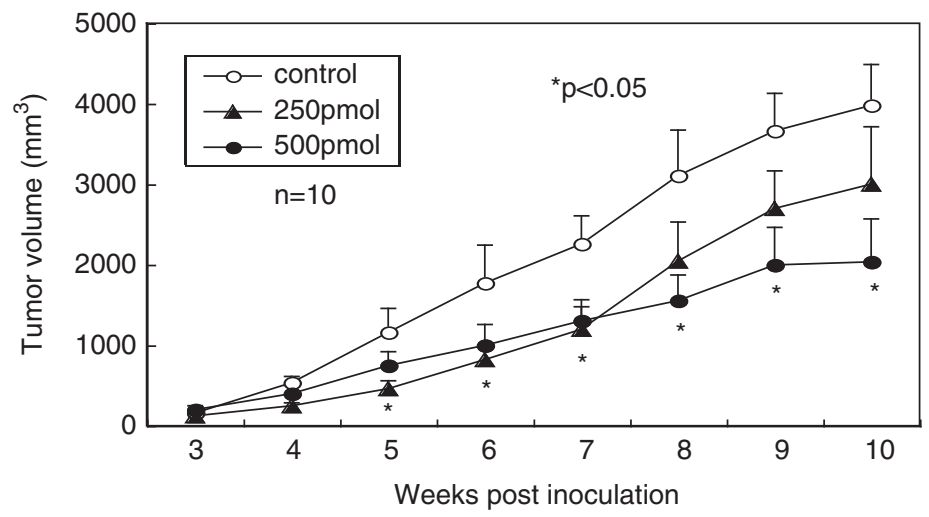

C

HE

Ki67

NEU3

TUNEL

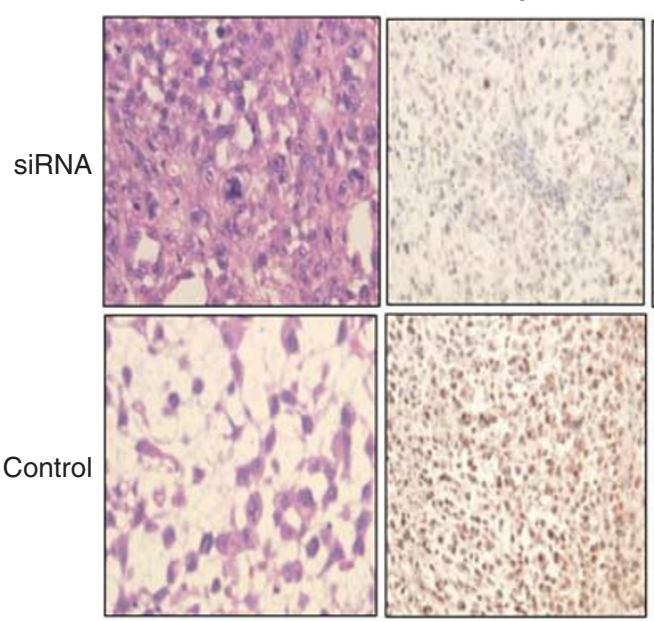

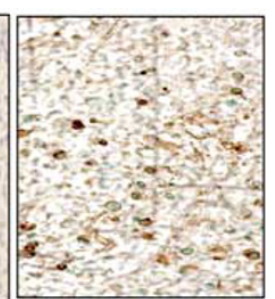

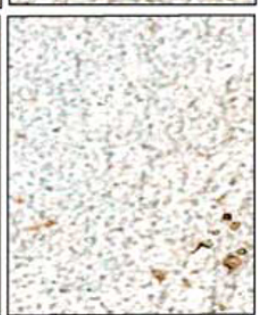

Figure 6 Suppression of prostate cancer growth in vitro and in vivo by treatment with NEU3 siRNA. (a) Reduction in the number of LNCaP and PC-3 cells by treatment with NEU3-specific siRNA, as assessed by MTT assays. (b) Reduction of the tumor volumes of xenografts implanted PC-3 cells by NEU3 siRNA treatment with atelocollagen. Different doses of NEU3 siRNA ( 0,250 or 500 pmol) were injected into three groups of athymic nude mice ( $n=10$ for each condition) and the tumor diameters were measured and compared as described in Materials and Methods. The statistical analyses were performed using the Kruska-Wallis test. (c) HE staining (left panel) and immunohistological staining of tumor tissues from the mice with anti-Ki-67 and -NEU3 antibodies (two middle panels). Detection of apoptosis by TUNEL method in the tissues was carried out using an apoptosis detection kit (right panel)

caspase-3 activation in HeLa cells. The siRNA treatment here reduced invasiveness and motility of cancer cells, and interestingly, did not affect normal cells, even if NEU3 levels were efficiently suppressed. Furthermore, NEU3 overexpression enhanced Ras activation and consequent stimulation of ERK and AKT, whereas the NEU3 siRNA inhibited Ras activity with downregulation of ERK and the AKT pathway. These results indicate that NEU3 has an important role in cancer cell survival, and its increased expression promotes malignancy probably by modulating Ras-mediated signaling.

In prostate cancer cells, consistent with other types of cancer, NEU3 activated the PI3K and MAPK pathways, 
associated with increase in mRNA and protein levels of growth factor receptors including EGFR and ERBB2 under androgen-deficient conditions. For development of hormonerefractory progression, it has been reported that enhanced AR mRNA and protein expression is a critical factor in conversion to a hormone-refractory state in the majority of patients who do not have AR mutations ${ }^{3}$ or amplification. ${ }^{4,5}$ Other mechanisms include cross-talk between ARs and other signaling pathways, perhaps mediated by growth factor receptors such as EGFR and ERBB2. In this context, NEU3 might again be a pivotal molecule acting to upregulate growth factor receptors. We do not know the detailed mechanism of the NEU3-mediated upregulation at present. However, our recent studies suggest the possibility that NEU3 undergoes transcriptional activation by SP1 transcription factor, ${ }^{29}$ which is positively regulated by several kinases including $\mathrm{ERK}^{32}$ and involved in transcriptional regulation of genes implicated in cell growth and tumorigenesis including EGFR. ${ }^{33}$ As proposed in Figure 5d, NEU3 upregulation may give rise to the increased expression of NEU3 itself and EGFR family by activating ERK and then SP1 in positive feedback loop. In addition to this transcriptional regulation, NEU3 may also positively regulate function of growth factor receptors probably through its association with the receptors and glycolipid changes as a result of its catalytic reaction. ${ }^{12}$ Our previous results showed that NEU3 changed glycolipid pattern, such as an accumulation of lactosylceramide in colon cancer, ${ }^{13}$ and in addition, NEU3 was co-immunoprecipitated with EGFR in HeLa cells. ${ }^{20}$ The examination of glycolipid changes by thinlayer chromatography in this study also revealed an increase in lactosylceramide in NEU3 overexpressing LNCap cells, although it is uncertain how the changes affect the growth factor receptors and it cannot be excluded the possibility of an involvement of other subtle glycolipid change.

In conclusion, our present observations strongly suggest that NEU3 is a pivotal molecule for regulation of progression of prostate cancer to androgen-independent growth. NEU3 might be used for the development of a new diagnostic approach capable of determining the malignant grade, and also as an excellent target for prostate cancer therapy by selective suppression of NEU3 using specific siRNAs.

\section{Materials and Methods \\ Patient samples. The specimens of tumor and non-tumor prostate tissues were obtained by transrectal needle biopsy from 34 patients diagnosed with prostate cancer at various clinical stages and 35 non-cancer patients including examples with benign hyperplasia. Histopathological findings of the needle biopsy specimens confirmed the diagnosis for all patients by examination of the specimens from the almost identical portion. Informed consent was obtained from each to allow use of the specimens for research purposes, and the study was approved by the Committee on Human Rights in Research at Miyagi Cancer Center.}

Cell lines and cell culture conditions. Human prostate cancer cell lines PC-3 (HSRRB, Osaka, Japan) and LNCaP-FGC (Cancer Cell Repository, Tohoku University, Sendai, Japan) were maintained in Ham F-12K containing $8 \%$ fetal bovine serum (FBS) and in RPMI 1640 containing 10\% FBS, respectively. To assess AR signaling, LNCaP cells were cultured in phenol-red free RPMI 1640 medium supplemented with charcoal-stripped $10 \%$ FBS for $24-48 \mathrm{~h}$, and treated with 1 or $5 \mathrm{nM}$ DHT (Sigma, St Louis, MO, USA) or $5 \mu$ M bicalutamide (Toronto Research Chemicals Inc., Toronto, ON, Canada) for $24 \mathrm{~h}$ before harvesting. In some experiments, LNCaP cells were incubated in RPMl 1640 medium with $50 \mu \mathrm{M}$ LY294002 and $20 \mu \mathrm{M}$ PD098059 (inhibitors of PI3K and MAPK, respectively) for $24 \mathrm{~h}$.
Transfections. For NEU3 overexpression, the entire open reading frame $(1.2 \mathrm{~kb})$ of the human NEU3 gene was inserted into a retrovirus vector pMXs-puro and the plasmid was introduced into PlatA, and target cells were then incubated with the culture media containing infectious viruses for two days and selected by cultivating in the presence of $1 \mu \mathrm{g} / \mathrm{ml}$ puromycin for 10-14 days as previously described. $^{34}$ siRNAs targeting NEU3 and control scrambled siRNA were synthesized by Dharmacon Inc. (Thermo Fisher Scientific, Rockford, IL, USA) as described. ${ }^{20}$ One of the siRNAs targeting NEU3 (no. 3) was $5^{\prime}$-AAGG GAGTGTGGTAAGTTT-3' beginning at nucleotide 839 of the NEU3 open reading frame sequence, and the scrambled control was $5^{\prime}$-GCGATTAATGTAGGTT CGA- $3^{\prime}$. The other siRNA targeting NEU3 synthesized by iGENE Therapeutics (Tsukuba, Japan) was $5^{\prime}$-GGTTACAGTAGAATGTGAAGTGGCA-3'. These two types of siRNA targeting NEU3 showed $75-95 \%$ NEU3 reduction and almost same results in various experiments, and therefore, the former was routinely utilized. siRNAs targeting AR(On-Target plus SMART pool L-003400), PIK3CA (siGENOME SMART pool M-003018), and MAP2K1 (siGENOME SMART pool M-003571) and a control siRNA (On-Target plus siControl Non-Targeting siRNA\#2) were from Dharmacon Inc (Thermo Fisher Scientific). Transfection was performed by nucleofection or with a Lipofectamine RNAiMAX reagent (Invitrogen, Carlsbad, $\mathrm{CA}, \mathrm{USA}$ ), and cells were used for experimentation at $24-48 \mathrm{~h}$ thereafter.

Sialidase activity assays. Crude extracts were used for sialidase assays using bovine brain gangliosides GM3 (Alexis Biochemicals, Lansen, Switzerland) as the substrate in the presence of Triton X-100 as described elsewhere. ${ }^{20}$ After incubation at $37^{\circ} \mathrm{C}$ for $10-30 \mathrm{~min}$, the amount of sialic acid released was determined by a modified thiobarbituric acid method or by fluorometric highperformance liquid chromatography with malononitrile. ${ }^{35}$ One unit of activity was defined as the amount of enzyme that cleaved $1 \mathrm{nmol}$ sialic acid from the substrates. Protein concentrations were determined by dye-binding assay (Bio-Rad Laboratories, Hercules, CA, USA).

Quantitative RT-PCR analysis. The mRNA levels of human NEU3 and other molecules were evaluated by quantitative RT-PCR (real-time PCR, Lightcycler, Roche Diagnostics) as described previously. ${ }^{20}$ Total RNA was isolated from biopsy specimens, as well as from PC-3 and LNCaP cells with an RNeasy kit (Qiagen, Hilden, Germany), and first strand cDNAs synthesized by reverse transcription were used as templates for PCR. Human NEU3 primers were 5'-AGGTCAGTCTCCAGTACCTTC-3' (forward) and 5'-ACATCCAGCATCCTGACT GTAG-3' (reverse). The others were: for keratin 17, $5^{\prime}$-GCTGGAGGTGAAGATCC GTGA-3' (forward) and $5^{\prime}$-ATTGTCCACGGTGGCTGTGA-3' (reverse); IL24, 5'-ACC CACAGCTATGCCTCTGATTG-3' (forward) and 5'-TGTTAAATTGGCGAAAGC AGCTC-3' (reverse); BCl-2, 5'-GTACGACAACCGGGAGATAGTGAT-3' (forward) and $5^{\prime}$-GCCTCCGTTATCCTGGATCCAGGT-3' (reverse); EGR-1, 5'-CAGGGCT TCGGACATGACA-3' (forward) and $5^{\prime}$-GACTTGGCTCTGAGAACCTCCATC-3' (reverse); AR, 5'-ACTTCACCGCACCTGATGTG-3' (forward) and 5'-TTCCGAAGAC GACAAGATGGA-3' (reverse); PSA, 5'-CAACCCTGGACCTCACACCTA-3' (forward) and $5^{\prime}$-GGAAATGACCAGGCCAAGAC-3' (reverse); EGFR, $5^{\prime}$-TGGTCCTTGGGA ATTTGGAA-3' (forward) and $5^{\prime}$-GGGCAATGAGGACATAACCAG-3' (reverse); ERBB2, 5'-TGGGAGCCTGGCATTTCTG-3' (forward) and 5'-TCCGGCCATGC TGAGATGTA-3' (reverse). To normalize for sample variation, human $\beta$-actin was assayed as an internal control. Densitometric analyses of PCR products were carried out with Quantity One 1D-analysis software (Bio-Rad Laboratories).

Immunoblotting. Cells were homogenized and solubilized in five volumes of ice-cold buffer ( $50 \mathrm{mM}$ Hepes (pH 7.5), $150 \mathrm{mM} \mathrm{NaCl}, 1 \%$ NP-40, $0.25 \%$ deoxycholate, $2 \mathrm{mM}$ EDTA, $10 \mathrm{mM} \mathrm{NaF}, 2 \mathrm{mM} \mathrm{Na}_{3} \mathrm{VO}_{4}, 2 \mathrm{mM} \mathrm{PMSF}, 7.5 \mu \mathrm{g} / \mathrm{ml}$ aprotinin and $10 \mu \mathrm{g} / \mathrm{ml}$ leupeptin) for immunoblotting. The cell lysates were separated on SDS-PAGE under reducing conditions, and analyzed by immunoblotting with the respective antibodies using ECL Plus Western blotting reagent (GE Healthcare UK Ltd., Buckinghamshire, UK). Antibodies for EGR-1 and PSA were from Santa Cruz (Santa Cruz, CA, USA) and for AR from Upstate. Anti-Akt, anti-phospho-Akt (Ser473), anti-p44/42 MAPK, anti-phosphop44/42 MAPK (Thr202/Tyr204) and anti-PI3K p110 $\alpha$ (C73F8) antibodies were from Cell Signaling Technology (Danvers, MA, USA), and anti-MEK1 antibody was from BD Biosciences (San Jose, CA, USA).

Thin-layer chromatography. Glycolipids were extracted from LNCaP cells as described elsewhere, ${ }^{14}$ fractionated by thin-layer chromatography on HPTLC 
plates (Baker, Phillipsburg, $\mathrm{NJ}$, USA) in $\mathrm{C} / \mathrm{M} / 0.5 \% . \mathrm{CaCl}_{2}(60: 40: 9, \mathrm{v} / \mathrm{v} / \mathrm{v})$ and visualized with orcinol- $\mathrm{H}_{2} \mathrm{SO}_{4}$.

Mouse models for tumor therapy. To generate tumor xenografts, $\mathrm{PC}-3$ cells $\left(6.0 \times 10^{6}\right)$ were injected subcutaneously in $100 \mu \mathrm{l}$ of serum-free F-12K medium through a 27-gauge needle into the lower flanks of 8-week-old athymic nude mice obtained from CLEA Japan Inc. (Tokyo, Japan). After 3-4 weeks when the tumor volume had reached at least $70-80 \mathrm{~mm}^{3}$, the tumor-bearing nude mice were treated with NEU3 siRNA with atelocollagen (Koken Co. Ltd, Tokyo, Japan) ${ }^{30,31}$ The concentration of atelocollagen was $1 \%$, and different doses $(0,250,500 \mathrm{pmol})$ of NEU3 siRNA (siSTABLE stability-enhanced oligonucleotide, Dharmacon Inc.) and one scrambled siRNA of the same type $(500 \mathrm{pmol})$ were administrated into the tumors two times every week. Tumor diameters were measured at maximum length and width with digital calipers, and the tumor volume was calculated by the ellipsoid formula: volume $=(\text { width })^{2} \times$ length $/ 2 .^{36}$ The data were statistically analyzed using the Kruskal-Wallis test for overall comparisons and the Tukey/Scheffe test for multiple pairwise comparisons. $P$-values $<0.05$ were considered to be significant. All animal experiments in this study were performed in compliance with the guidelines of Laboratory Animal Research, Miyagi Cancer Center Research Institute.

Immunohistochemistry. Removed tissues were fixed in $10 \%$ neutral buffered formaldehyde 7 days, routinely processed for embedding in paraffin, and sectioned $2.5 \mathrm{~mm}$ thickness. To assess NEU3 and ki-67 expression, the sections were pretreated for antigen recovery by heating in $0.01 \mathrm{M}$ citrate $(\mathrm{pH} 6.0)$ in microwave oven, and incubated with the anti-NEU3 monoclonal antibody prepared previously ${ }^{37}$ and an anti-Ki67 antibody (Dako Cytomation, Dako, Glostrup, Denmark), respectively. Determination of cell apoptosis in tumor tissues was performed using an ApopTag plus peroxidase in situ apoptosis detection kit (Chemicon International Inc., Hants, UK) according to the manufacturer's instructions.

\section{Conflict of Interest}

The authors declare no conflict of interest.

Acknowledgements. We are grateful for Dr. Ochiya T (National Cancer Center, Tokyo) for his helpful suggestion for usage of atelocollagen. This work was supported in part by CREST of Japan Science and Technology Agency and by Grants-in Aid (on priority areas 20013047) for Scientific Research on Priority Areas in Cancer from the Ministry of Education, Culture, Sports, Science and Technology of Japan.

1. Heinlein CA, Chang C. Androgen receptor in prostate cancer. Endocr Rev 2004; 25 : 276-308

2. Chen CD, Welsbie DS, Tran C, Baek SH, Chen R, Vessella R et al. Molecular determinants of resistance to antiandrogen therapy. Nat Med 2004; 10: 33-39.

3. Taplin ME, Bubley GJ, Shuster TD, Frantz ME, Spooner AE, Ogata GK et al. Mutation of the androgen-receptor gene in metastatic androgen-independent prostate cancer. $N E n g / J$ Med 1995; 332: 1393-1398.

4. Linja MJ, Savinainen KJ, Saramaki OR, Tammela TL, Vessella RL, Visakorpi T. Amplification and overexpression of androgen receptor gene in hormone-refractory prostate cancer. Cancer Res 2001; 61: 3550-3555.

5. Edwards J, Krishna NS, Grigor KM, Bartlett JM. Androgen receptor gene amplification and protein expression in hormone refractory prostate cancer. Br J Cancer 2003; 89: 552-556.

6. Bakin RE, Gioeli D, Sikes RA, Bissonette EA, Weber MJ. Constitutive activation of the Ras/ mitogen-activated protein kinase signaling pathway promotes androgen hypersensitivity in LNCaP prostate cancer cells. Cancer Res 2003; 63: 1981-1989.

7. Rahman M, Miyamoto $\mathrm{H}$, Chang $\mathrm{C}$. Androgen receptor coregulators in prostate cancer: mechanism and clinical implications. Clin Cancer Res 2004; 10: 2208-2219.

8. Abdulkadir SA, Qu Z, Garabedian E, Song SK, Peters TJ, Svaren J et al. Impaired prostate tumorigenesis in Egr1-defficient mice. Nat Med 2001; 7: 101-107.

9. Yang S-Z, Eltoum IA, Abdulkadir SA. Enhanced EGR-1 activity promotes the growth of prostate cancer cells in an androgen-deleted environment. J Cell Biochem 2006; 97: 1292-1299.

10. Yang S-Z, Abdulkadir SA. Early growth response gene1 modulates androgen receptor signaling in prostate carcinoma cells. J Biol Chem 2003; 278: 39906-39911.
11. Miyagi T, Wada T, Yamaguchi K. Review: roles of plasma membrane- associated sialidase NEU3 in human cancers. Biochim Biophys Acta 2008; 1780: 532-537.

12. Miyagi T, Wada T, Yamaguchi K, Hata K, Shiozaki K. JB minireview: plasma membraneassociated sialidase as a crucial regulator of transmembrane signalling. J Biochem 2008; 144: $279-285$

13. Kakugawa $\mathrm{Y}$, Wada $\mathrm{T}$, Yamaguchi $\mathrm{K}$, Yamanami $\mathrm{H}$, Ouchi $\mathrm{K}$, Sato I et al. Up-regulation of plasma membrane- associated ganglioside sialidase (Neu3) in human colon cancer and its involvement in apoptosis suppression. Proc Natl Acad Sci USA 2002; 99: 10718-10723.

14. Ueno S, Saito S, Wada T, Yamaguchi K, Satoh M, Arai $Y$ et al. Plasma membraneassociated sialidase is up-regulated in renal cell carcinoma and promotes the interleukin-6 induced apoptosis suppression and cell motility. J Biol Chem 2006; 281: 7756-7764.

15. Nomura H, Tamada $Y$, Miyagi T, Suzuki A, Taira M, Suzuki N et al. Exression of NEU3 (plasma membrane-associated sialidase) in clear cell adenocarcinoma of the overy: its relationship with T factor of pTNM classification. Oncol Res 2006; 16: 289-297.

16. Hakomori S. Glycosylation defining cancer malignancy: new wine in an old bottle. Proc Nat/ Acd Sci USA 2002; 99: 10231-10233.

17. Varki NM, Varki A. Diversity in cell surface sialic acid presentations: implications for biology and disease. Lab Invest 2007; 87: 851-857.

18. Lau KS, Dennis JW. N-glycans in cancer progression. Glycobiology 2008; 18: 750-760.

19. Miyagi T. Aberrant expression of sialidase and cancer progression. Proc Jpn Acad, Ser B Phys Biol Sci 2008; 84: 407-418.

20. Wada T, Hata K, Yamaguchi K, Shiozaki K, Koseki K, Moriya S et al. A crucial role of plasma membrane-associated sialidase in the survival of human cancer cells. Oncogene 2007; 26: 2483-2490.

21. Kato K, Shiga K, Yamaguchi K, Hata K, Kobayashi T, Miyazaki K et al. Plasma membraneassociated sialidase (NEU3) differentially regulates integrin-mediated cell proliferation through laminin- and fibronectin-derived signaling. Biochem J 2006; 394: 647-656.

22. Ravindranath $\mathrm{MH}$, Muthugounder $\mathrm{S}$, Presser $\mathrm{N}, \mathrm{Ye} \mathrm{X}$, Brosman $\mathrm{S}$, Morton $\mathrm{DL}$. Endogenous immune response to gangliosides in patients with confined prostate cancer. Int J Cancer 2005; 116: 368-377.

23. Manin M, Baron S, Goossens K, Beaudoin C, Jean C, Veyssiere G et al. Androgen receptor expression is regulated by the phosphoinositide 3-kinase/Akt pathway in normal and tumor epithelial cells. Biochem J 2002; 366: 729-736.

24. Craft N, Shostak Y, Carey M, Sawyers CL. A mechanism for hormone-independent prostate cancer through modulation of androgen receptor signaling by the HER-2/neu tyrosine kinase. Nat Med 1999; 5: 280-285.

25. Gregory CW, Fei X, Ponguta LA, He B, Bill HM, French FS et al. Epidermel growth factor increases coactivation of the androgen receptor in recurrent prostate cancer. J Biol Chem 2004; 279: 7119-7130.

26. Berger R, Lin DI, Nieto M, Sicinska E, Garraway LA, Adams H et al. Androgen-dependent regulation of Her-2/neu in prostate cancer cells. Cancer Res 2006; 66: 5723-5728.

27. Pignon J-C, Koopmansch B, Nolens G, Delacroix L, Waltregny D, Winkler R. Androgen receptor controls EGFR and ERBB2 gene expression at different levels in prostate cancer cell lines. Cancer Res 2009; 69: 2941-2949.

28. Cai C, Portnoy DC, Wang H, Jiang X, Chen S, Balk SP. Androgen receptor expression in prostate cancer cells is suppressed by activation of epidermal growth factor receptor and ErbB2. Cancer Res 2009; 69: 5202-5209.

29. Yamaguchi K, Koseki K, Shiozaki M, Shimada Y, Wada T, Miyagi T. Regulation of plasma membrane-associated sialidase NEU3 gene by Sp1/Sp3 transcription factors. Biochem J 2010; 430: 107-117.

30. Ochiya T, Takahama Y, Nagahara S, Sumita Y, Hisada A, Itoh $\mathrm{H}$ et al. New delivery system for plasmid DNA in vivo using atelocollagen as a carrier material: the Minipellet. Nat Med 1999; 5: 707-710.

31. Takeshita F, Minakuchi Y, Nagahara S, Honma K, Sasaki H, Hirai K et al. Efficient delivery of small interfering RNA to bone-metastatic tumors by using atelocollagen in vivo. Proc Natl Acad Sci USA 2005; 102: 12177-12182.

32. Wierstra I. Sp1: emerging roles - beyond constitutive activation of TATA-less housekeeping genes. Biochem Biophys Res Commun 2008; 372: 1-13.

33. Safe $\mathrm{S}$, Abdelrahim M. Sp transcription factor family and its role in cancer. Eur J Cancer 2005; 41: 2438-2448.

34. Kitamura T, Koshino Y, Shibata F, Oki T, Nakajima H, Nosaka T et al. Retrovirus-mediated gene transfer and expression cloning: powerful tools in functional genomics. Exp Hematol 2003; 31: 1007-1014.

35. Li K. Determination of sialic acids in human serum by reversed-phase liquid chromatography with fluorimatric detection. J Chromatogr 1992; 579: 209-213.

36. Burfeind P, Chernicky CL, Rininsland F, Ilan J, llan J. Antisense RNA to the type I insulinlike growth factor receptor suppresses tumor growth and prevents invasion by rat prostate cancer cells in vivo. Proc Natl Acad Sci USA 1996; 93: 7263-7268.

37. Wang Y, Yamaguchi K, Wada T, Hata K, Zhao X, Fujimoto T et al. A close association of ganglioside-specific sialidase, Neu3, with caveolin in membrane microdomains. J Biol Chem 2002; 277: 26252-26259. 